\title{
Factors Predicting Sexual Risk Behaviors of Adolescents in North-Eastern Thailand
}

\author{
Athiwat BUTDABUT ${ }^{1}$ and Pissamai HOMCHAMPA \\ Health System Science Department,Faculty of Medicine, Mahasarakham University, \\ Maha Sarakham, Thailand
}

\begin{abstract}
The number of young people who have had sex at an early age increases in proportion, it concerns unsafe sexual behaviors, teenage pregnancy, HIV aids and sexually transmitted infections (STIs). This study examines the health behaviors and factors predicting sexual risk behaviors pertaining to teenage pregnancy among adolescents in Thailand. Adolescents consulted the reproductive health center about problems with the same gender. The factors of adolescent reproductive behaviors were significantly associated with age, education level, and the perception of peer norms. Receiving social support from media information also significantly correlated with those behaviors. The results recommend that to prevent premature pregnancy, adolescents should protect themselves. Parents should take the issue of social media use by their teenagers very seriously.
\end{abstract}

Keywords: Teen pregnancy, teen sexual health, pregnancy prevention, reproductive health

\section{Introduction}

Adolescence is a unique age of transition from childhood to adulthood in many aspects [7]. The subjects change many varieties including physical, mental, and social shifts. These changes bring both risks and opportunities that influence the development into adulthood. Teenagers today are growing up in societies with developed economies, social systems and technologies, especially communication technology. Advancements such as social media with mobile phones, tablets, and other wireless devices have revolutionized communication styles and the way of accessing knowledge. According to WHO, approximately 12 million girls aged 15-19 years and at least 777,000 girls under 15 years give birth each year in developing regions [1]. At least 10 million unintended pregnancies occur each year among adolescent girls aged 15-19 years in the developing world [5]. Complications during pregnancy and childbirth are the leading cause of death for 15-19-year-old girls globally [6]. Adolescent mothers (ages 10-19 years) face higher risks of eclampsia, puerperal endometritis, and systemic infections, compared to women aged 20 to 24 years, and their babies face higher risks of low birth weight, preterm delivery and severe neonatal conditions [10]. This study investigates the behaviors that may play a role in the reproductive health of teenagers in Health Region 7 in Northeast of Thailand, aiming at determining which behaviors enhance reproductive health

1 Corresponding Author: Athiwat Butdabut, Faculty of Medicine, Mahasarakham University, MahaSarakham, Thailand. Email: athiwatbutdabut@gmail.com 
promotion among teens with the goal of further developing, supporting, and encouraging them.

\section{Methods}

\subsection{Study Design and Participants}

The sample comprised 15-19 years old secondary school teenagers from 4 provinces under the responsibility of the 7th Regional Health Promotion Centre including Khon Kean, Roi-Et, Mahasarakham, and Kalasin. Selected by a randomization method, we included 389 teenagers in our study.

\subsection{The Questionnaire Ethical Concern and Statistical Analysis}

The questionnaire consisted of 137 questions on following topics: 1) Socio-economic and characteristic, 2) behaviors related to reproductive health, 3) attitudes towards selfcontrol ability, 4) gender, 5) peer norms perception, 6) social support in media information, confirmed by Index of Item-Objective Congruency (IOC) scores $\geq 0.5$ Cronbach's $\alpha \geq 0.7$. The participants were protected, being very aware of the individual rights and taking action to prevent inadvertent negative effects based on ethical research principles, accredited by the Human Research Ethics Committee of the Mahasarakham University (No. 78/2018). Data analysis used descriptive statistics (Pearson Chi-square), then testing correlation of each categorical data by $\chi^{2}$ test. The level of statistical significance was $\mathrm{p}<0.05$. All data were analyzed by SPSS Statistics version 18 .

\section{Results}

Table 1. Correlation between other Factors and Teen Reproductive Health Behavior $(\mathrm{n}=389)$

\begin{tabular}{|c|c|c|c|c|c|}
\hline \multirow{2}{*}{ Factors } & \multicolumn{3}{|c|}{ Adolescent Reproductive Behaviors } & \multirow{2}{*}{$\begin{array}{l}\text { Chi- } \\
\text { Square }\end{array}$} & \multirow{2}{*}{$p$-value } \\
\hline & Poor & Medium & Good & & \\
\hline Gender & & & & $8.489^{\mathrm{a}}$ & 0.075 \\
\hline - Male & $17(25.6)$ & $28(35.4)$ & $34(43.0)$ & & \\
\hline - Female & $56(19.6)$ & $139(48.8)$ & $90(31.6)$ & & \\
\hline - $\quad$ LGBT & $7(28.0)$ & $14(56.0)$ & $4(16.0)$ & & \\
\hline Age & & & & $27.217^{\mathrm{a}}$ & $<0.001^{* *}$ \\
\hline - Less than 16 years & $12(13.3)$ & $28(31.1)$ & $50(55.6)$ & & \\
\hline - Over 16 years & $68(22.7)$ & $153(51.2)$ & $78(26.1)$ & & \\
\hline Education Level & & & & $47.366^{\mathrm{b}}$ & $<0.001^{* *}$ \\
\hline - $\quad$ High school & $22(12.0)$ & $71(38.8)$ & $90(49.2)$ & & \\
\hline - Voc. Cert. & $55(28.1)$ & $103(52.6)$ & $38(19.3)$ & & \\
\hline High Voc. Cert. & $3(30.0)$ & $7(70.0)$ & $0(0.0)$ & & \\
\hline GPA & & & & $3.544^{\mathrm{a}}$ & 0.170 \\
\hline - $\quad$ Less than 3.00 & $12(32.4)$ & $15(40.6)$ & $10(27.0)$ & & \\
\hline - $\quad$ More than or equal to 3.00 & $68(19.3)$ & $166(47.2)$ & $118(33.5)$ & & \\
\hline Monthly Expense & & & & $1.737^{\mathrm{a}}$ & 0.420 \\
\hline - Less than2,000 baht & $24(24.7)$ & $45(46.4)$ & $28(28.9)$ & & \\
\hline - $\quad$ More than 2,000 baht & $56(19.2)$ & $136(46.6)$ & $100(34.2)$ & & \\
\hline Peer Norms Perception & & & & $80.233^{\mathrm{a}}$ & $<0.001 * *$ \\
\hline - Very risky level & $69(30.3)$ & $115(50.4)$ & $44(19.3)$ & & \\
\hline - $\quad$ Moderate risk level & $7(16.3)$ & $26(60.5)$ & $10(23.2)$ & & \\
\hline - $\quad$ Low risk level & $4(3.4)$ & $40(33.9)$ & $74(62.7)$ & & \\
\hline $\begin{array}{l}\text { Receiving Social Support Information } \\
\text { from Media }\end{array}$ & & & & $11.468^{\mathrm{b}}$ & $0.013^{*}$ \\
\hline - Internet/TV programme & $68(18.8)$ & $170(47.1)$ & $123(34.1)$ & & \\
\hline - Printed media & $10(50.0)$ & $8(40.0)$ & $2(10.0)$ & & \\
\hline - Health personnel & $2(25.0)$ & $3(37.5)$ & $3(37.5)$ & & \\
\hline
\end{tabular}


The gender distribution among the adolescent showed females $(73.3 \%)$, males $(20.3 \%)$, and LGBT (Lesbian, Gay, Bisexual, and Transgender) (6.4\%) with an average age of $16.55 \pm 1.18$. Most of the students studied at the first year vocational certificate level $(27.0 \%)$. The most family received an income less than 15,000 baht per month $(36.4 \%)$. Most teen caregiver's relationships were parents (76.9). Most people lived in parent's house $(88.9 \%)$, mostly in adolescent's family with 4-6 people (68.6\%). Adolescents and friends consulted on reproductive health problems mostly hold the same gender (88.9). The factors significantly associated with adolescent reproductive behaviors were: 1) age group ( $p$-value $<0.001), 2$ ) educational level ( $p$-value $<0.001), 3)$ perception of peer group norms $(p$-value $<0.001)$, and 4$)$ social support in media information $(p$-value $=$ $0.013)$.

\section{Discussion}

\subsection{Characteristics of the Adolescent and their Reproductive Behaviors}

The result revealed that age group and educational level were significantly associated with adolescent reproductive behaviors. An earlier study reported that the age group is of relevance. Females who reported indirect aggression toward peers had earlier ages at first sexual intercourse, while females who were more victimized in adolescence experienced later ages at first sexual intercourse [9]. The education also affected several things for females in Pakistan. Literacy, for instance, is lower for women than for men. Only $20 \%$ of all females have attended primary school. Although most women know at least 1 contraceptive method, it is the urban educated woman who is twice as likely to know a source of supply and 5 times more likely to be a user [2]. Thai culture values virginity in the female gender, and marriage is the method that makes early pregnancy more acceptable. The research results eventually prompted health officials to advise teenagers on pregnancy prevention. Most of the peer norms perception are at high and medium risk level regarding adolescent reproductive behaviors. Van de Bongardt et al. [8] performed a meta-analysis to investigate the associations between three types of peer norms (descriptive norms (peer sexual behaviors), injunctive norms (peer sexual attitudes)), the peer pressure to have sex, and two adolescent sexual behavior outcomes (sexual activity and sexual risk behavior). Adolescent sexual activity was stronger associated with descriptive norms than with injunctive norms or peer pressure. Compared with the sexual activity outcome, the effect size of descriptive norms (peer sexual risk behavior) for sexual risk behavior was smaller. They also approved that age, gender, peer type, and socio-cultural context significantly moderated these associations. Another study explains that perceived peer norms supporting safer sex were inversely associated with recently having two or more sexual partners after controlling for demographic characteristics. Perceived peer norms around safer sexual behavior contribute to a lower likelihood of engaging in two HIV/STI risk behaviors: inconsistent condom use and multiple partnering [4].

\subsection{Social Media Support Information}

The adolescent's behaviors are also correlated to social support received by gathering information from any kinds of media. Regarding the use of information especially in the 
context of health education such as safe sex, we recommend that adults should select supporting media. A study [3] conducted a systematic review of the literature to examine the effectiveness of social media among young adults aged 15 through 24 years, which indicated that social media and text messaging can increase knowledge regarding the prevention of STDs. These interventions may also affect behavior, such as screening/testing for STDs, sexual risk behaviors, and STD acquisition, but the evidence for effect is weak.

\section{Conclusion}

In conclusion, age group and educational level were significantly associated with adolescent reproductive behaviors. It also verified that the adolescent is influenced by peer norms perceptions in both the very risk level and medium level of adolescent reproductive behaviors. These findings emerge empirical factors for the risk behavior especially of peer norms and hold important implications for reproductive health of teenagers.

\section{References}

[1] Darroch JE, Woog V, Bankole A, Ashford LS. Adding it up: costs and benefits of meeting the contraceptive needs of adolescents. New York: Guttmacher Institute, 2016, https://www.guttmacher.org/report/adding-it-meeting-contraceptive-needs-....

[2] Greenspan A. Effects of education on reproductive behavior: lessons from Pakistan. Asia Pac Pop Policy. 1992 Dec;(23):1-4.

[3] Jones K, Eathington P, Baldwin K, Sipsma H. The impact of health education transmitted via social media or text messaging on adolescent and young adult risky sexual behavior: a systematic review of the literature. Sex Transm Dis. 2014 Jul;41(7):413-9. doi: 10.1097/OLQ.0000000000000146.

[4] Kapadia F, Frye V, Bonner S, Emmanuel PJ, Samples CL, Latka MH. Perceived peer safer sex norms and sexual risk behaviors among substance-using Latino adolescents. AIDS Education and Prevention 01 Feb 2012, 24(1):27-40. DOI: 10.1521/aeap.2012.24.1.27 PMID: 22339143

[5] Kiani MA, Ghazanfarpour M, Saeidi M. Adolescent pregnancy: a health challenge. International Journal of Pediatrics 2019; 7(7): 9749-9752.

[6] Neal S, Matthews Z, Frost M, Fogstad H, Camacho AV, Laski L. Childbearing in adolescents aged 1215 years in low resource countries: a neglected issue. New estimates from demographic and household surveys in 42 countries. Acta Obstet Gynecol Scand. 2012 Sep;91(9):1114-8. doi: 10.1111/j.16000412.2012.01467.x.

[7] Spear LP. Neurobehavioral changes in adolescence. Current Directions in Psychological Science 2000; 9(4): 111-114.

[8] Van de Bongardt D, Reitz E, Sandfort T, Deković M. A meta-analysis of the relations between three types of peer norms and adolescent sexual behavior. Pers Soc Psychol Rev. 2015 Aug;19(3):203-34. doi: $10.1177 / 1088868314544223$.

[9] White DD, Gallup AC, Gallup Jr GG. Indirect peer aggression in adolescence and reproductive behavior. Evolutionary Psychology 2010; 8: 147470491000800106.

[10] WHO U, Mathers C. Global strategy for women's, children's and adolescents' health (2016-2030). Organization 2016; 201: 4-103. 